\title{
Administrative and Judicial (Penal) Measures Taken by The Algerian Authorities to Confront the Coronavirus
}

\author{
Aicha Abd Elhamid \\ Faculty of Law and Political Sciences, University of Chadli Ben Jadid, Al-Tarif, Algeria \\ malekcaroma23@gmail.com
}

\begin{abstract}
:
In the face of the Corona pandemic, like the countries of the world, Algeria has taken precautionary and other deterrent measures that keep pace with and accompany the epidemic, aiming to protect the security and safety of the citizen in the first place. Which was approved by the President of the Republic, Abdel Majid Tebboune, and aimed at preventing and combating the spread of the Corona epidemic (Covid 19), as Executive Decree No. 20-69 issued on March 21, 2020, came with measures and measures that ranged from approving a total quarantine for some states, then Gradually, the state entered a partial quarantine for all the states of the homeland, with a variation in the hours of quarantine. And in front of the quarantine procedures, to ensure the safety and security of the citizen and to preserve public order within society, and in order to respect the quarantine procedures, Algeria entered into deterrent measures, ranging between Administrative penalties and criminal penalties imposed on citizens, as well as the adoption of deterrent measures for all violators of the quarantine procedures, and these penalties were financial fines and imprisonment as a penalty depriving freedom, as the Algerian legislator amended the Penal Code. Irrigation including articles stipulating criminal penalties for all quarantine violators and for everyone who stands an obstacle to the application of quarantine measures and the preservation of public order by the police and gendarmerie agents to maintain security.
\end{abstract}

Keywords: Quarantine; Administrative Procedures; Judicial Procedures; Corona; Penal Code. 


\section{REFERENCES:}

Abdullah Al-Zayt, Montaser, Abdullah Al-Zuzi, Mu'tasim, Managing the economic crisis in light of the Corona epidemic according to the principles of Islamic economics, International Islamic Economics Journal, April, 2020 AD = 1441 AH, Issue 95, pp. 56-64.

Belaidoun, Awad, Mechanisms for maintaining work positions in light of the Corona pandemic crisis, Journal of Labor and Employment Law, June $2020 \mathrm{AD}=1441 \mathrm{AH}$, Volume 6, Issue 1, pp. 37-91.

Jaloud, Amer Muhammad Nizar, The Jurisprudence of Epidemiology, Kay Publishing House, 2020 AD = 1441 AH, p.11.

Balasri, to Hussein, Sharia rules for allocating necessary resources and goods in light of exceptional circumstances, the Corona crisis as a model, International Islamic Economics Journal, April 2020 $\mathrm{AD}=1441 \mathrm{AH}$, Issue 95, pp. 66-76.

Algerian Army Journal, issue 681 for April $2020=1441$ AH.

The Algerian News Agency websitewww.aps@dz

See Article 10 of Law No. 04-20 of December 25, 2004 related to the prevention of major dangers and the management of disasters in the context of sustainable development, JR No. 84, dated December 29, $2004 \mathrm{AD}=1424 \mathrm{AH}$.

Gharbi, Azzouz, Disaster Management and Major Risks in Algeria in the Light of Law 04-20, Journal of Legal Studies and Research, January $2020 \mathrm{AD}=1441 \mathrm{AH}$, Volume 04, Issue 02, pp. 49-56.

Souria, Sharif, The Legal Framework for Natural Disaster Management in Algeria, Professor Al-Bahith for Legal and Political Studies, March 2019 AD = 1440 A.H., University of Messila, Algeria, Issue 9, p. 365.

Sophie, Mohamed, Television Media and Managing Natural Disasters, Analysis of the Content of the Television Image in the Seismic "Idols" and "Boumerdes", Master's Memorandum, University of Oran, 2011 $\mathrm{AD}=1432 \mathrm{AH}, \mathrm{p} .57$.

Baghdadi, Faisal, The Role of Leadership in Crisis Management in the Region, Master Memorandum, Faculty of Law, University of Messila, 2014 AD $=1435$ AH, p. 3.

F. Royer collard cité par j. Gicquel, Essai sur la pratique de la 5éme république, Paris, L.G.D.J, 1968, P28.

Mouloud, Mansour, Research in Constitutional Law, ENAG House, 2015 AD = 1436 AH, p. 108.

Executive Decree 20-69, Official Gazette No. 15 of March 21, 2020 AD = 1441 AH.

Boualchair, Said, Powers of the President of the Republic in Extraordinary Circumstances, Algerian Journal of Legal and Political Sciences, $2013 \mathrm{AD}=1434 \mathrm{AH}$, Issue 2, p. 21.

Intervention of Dr. Aisha Abdel-Hamid at a national forum on crises and disasters at the University of May 08, 1945 in Qalma, on December 16, 2019 AD = 1440 AH, the title of the intervention: Humanitarian intervention of the People's National Army during the Chlef earthquake (idols) 1980 ..

Al-Ashawi, Abdel Aziz, Human Rights in International Law, p. 421, Khaldounia House, 2011 AD = 1432 AH, Algeria.

Article 02 of Law No. 91-23 of 29 Jumada Al-Ula 1412, corresponding to December 6, 1991, related to the contribution of the People's National Army to the tasks of protecting public security outside of exceptional cases, Official Gazette of the Algerian Republic, No. 63, issued on December 06, 1991.

Jabbar, Salah al-Din, Military Judiciary in Algerian Legislation and Comparative Law, Dat, p. 277, Dar Khaldounia, $2010 \mathrm{AD}=1431 \mathrm{AH}$, Algeria.

Tabash, Ezz El-Din, Explanation of the Special Section of the Penal Code, Crimes Against Persons and Money, pp. 45-46, Dar Belqis, Algeria, $2018 \mathrm{AD}=1439 \mathrm{AH}$.

Official Gazette No. 25 dated April 29, 2020 AD = 1441 AH.

Al-Jaish Magazine, file issue, Criminalization of Acts That Threaten the Security and Stability of the Country, Issue 682 issued in May $2020 \mathrm{AD}=1441 \mathrm{AH}$.

Ohaibi, Abdallah, Explanation of the Algerian Penal Code, General Section, p. 260, ENAG House, 2018 CE, $1439 \mathrm{AH}$, Algeria. 
Article 9, Paragraph 01 of Ordinance 66-156 of June 1966 containing the amended and supplemented penal code.

Article 253 bis 5 of Law 20-66.

Official Gazette Issue 25 dated April 29, $2020 \mathrm{AD}=1441 \mathrm{AH}$.

See Ordinance 66-155 of June 8, 1966 containing the amended and supplemented Criminal Procedure Law. 


\title{
الإجر اءات الإدارية والقضائية (الجزائية) التي اتخذنها السلطات الجزائرية لمجابهة فيروس
}

\author{
كورونا \\ عائشة عبد الحميد \\ كلية الحقوق و العلوم السياسية، جامعة الشاذلي بن جديد، الطارف، الجزائر
}

ملخص البـحث

قامت الجزائر و في مواجهة جائحة كورونا على غرار دول العالم ، بإجراءات احترازية و اخرى ردعية مواكبة و ملازمة للوباء ، هدفها حماية امن وسلامة المواطن بالدرجة الاولى ، حيث صدر في العدد الاخير من الجريدة الرسمية الجزائرية لشهر مارس ، المرسوم الذي يحدد و يوضح كيفيات تطبيق الاجر اءات التي اقرها رئيس الجمهورية عبد المججيد تبون ، و الرامية الى الوقاية من انتشار وباء كورونا ( كوفيد 19) و مكافحته ، حيث جاء المرسوم التنفيذي رقم 20-69 الصادر في 21 مارس 2020 ، 20 بتدابير و اجراءات تراوحت ما بين اقرار الحجر الكلي لبعض الولايات، ثم تدريجيا دخلت الدولة في حجر جزئي لكل ولايات الوطن ، مع تفاوت في ساعات الحجر .و امام اجر اءات الحجر ، و ضمانا لسلامة و امن المواطن و حفاظا على النظام العام داخل المجتمع ، و من اجل احترام اجراءات الحجر ، دخلت الجزائر في اجراءات ردعية ، تراوحت بين العقويات الادارية و العقوبات الجزائية المفروضة على المواطنين، و كذلك اقرار اجراءات رادعة لكل المخالفين لإجراءات الحجر ، و هذه العقوبات كانت الغر امة المالية ، و الحبس كعقوبة سالبة للحرية. حيث قام المشرع الجزائري بتعديل قانون العقويات الجزائري مضمنا اياه مواد تقر عقوبات جزائية لكل مخالفي الحجر و لكل من يقف عائقا امام تطبيق اجر اءات الحجر و المحافظة على النظام العام من قبل اعوان المحافظة على الامن من شرطة و درك.

الكلهات المفتاحية: الحجر الصحي، الإجراءات القضائية، كورونا، قانون العقوبات، الإجراءات الإداريـة.

Crossref doi https://doi.org/10.51345/.v31i1.218.g150 


\section{المقدمة}

في إطار الإجراءات التي اتخذتها بلادنا لمواجهة وباء "كورونا" و تكملة للإجراءات المنصوص عليها في المرسوم التنفيذي رقم 20-69 الصادر في 21 مارس 2020، قد أتبع بالمرسوم التنفيذي رقم 20-0 70 لمجابهة فيروس كورونا 190 2، أوعدو البشرية الخفي الذي بات يشكل تهديدا كبيرا على حياة البشرية بسبب السرعة الفائقة لانتشار عدواه من شخص لآخر. فبعد أن تم إطلاق تسمية "كوف المستجد 2019 " على فيروس كورونا في المر احل الأولى من ظهوره، تم تغيير التسمية مرة أخرى لتصبح "سارس - كوف2" سارس هي: (متلازمة الالتهاب الرئوي) وكوف تعني "كورونا فيروس" و بتاريخ 11 فيفري 2020 سمي الفيروس كوفيد 19، حيث أعلنت منظمة الصحة العالمية بتاريخ 30 جانفي 200 2، ظرفا صحيا استعجاليا عالميا نتيجة إعادة ظهور فيروس خطير و بتاريخ 11 فيفري سمي المرض الناجم عن هذا الفيروس المستجد "كوفيد 19" لتصنف المنظمة العالمية للصحة بتاريخ 11

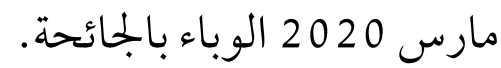
رصدت الجزائر لمو اجهة الفيروس و على غرار باقي الدول عدة إجراءات هناك الإدارية منها و هناك الجنائية. و على ما تقدم يمكن طرح الإشكالية التالية: كيف عالج المشرع الجزائري أزمة كورونا على مستوى الضبط الإداري؟ و تقييده للحريات من جهة ، و ما هي أوجه العقوبات الردعية التي جاء بها قانون العقوبات المعدل و المتمم؟

يشهد العالم اليوم أزمة ويائية شرسة، شلت أعمالهم وأنقصت أعدادهم وأموالهم وأصابت شتى ججالات حياتهم، وما بأيديهم سوى تسخير الجهود و الطاقات في سبيل الوقوف في وجهرا(1)، بعد أن تم تكيف فيروس كورونا كوفيد 19 كوباء عالمي من طرف منظمة الصحة العالمية(2). 
ما إن تحل أزمة من الأزمات على المجتمعات والبلدان حتى بين الأفر اد ، فعلى سبيل المثال فمنذ إنتشار فيروس ( كوفيد-19) في خختلف الأصقاع والأمصار، فزع الناس وتوجسوا صيفة، لا لكون الفيروس فتاكا وقاتلا فحسب بل لكون الاثر الذي أسدله على الإقتصاد العام في العالم ، وهلع خاص من المرضى ويثاره المسببة بالموت المنعزل من غير أهل ولأحبة، وخوف آخر من خلال الإقدام على التموين الذاتي والمنزلي والزيادة في شراء الحاجيات الغذائية والدوائية(3). فمها تضافرت جهود السلطات المختصة بالمراقبة والزجر، وتسجيل المخالفات، وتوفير جميع الإمكانات البشرية واللوجستية وإسقاط الضرائب ودعم الشركات لضبط السوق، تبقى سلطة الضمير والأخلاق واستحضار القيم الشرعية، من أهم ما يمكن أن نتجاوز به مثل هذه السلوكات فتخفف المؤونة ويكون الاهتمام منصبا بالأساس على تجاوز المشكلة الاصل (4). وبتاريخ 11 فيفري 2002 سمي هذا المرض الناجم عن هذا الفيروس المستجد كوفيد 19، لتصنفه المنظمة العالمية للصحية في 11 مارس 2020 الوباء بالجائحة(5). منذ بداية الأزمة الصحية المعروفة بكورونا أو كوفيد 19 اتخذت الإدارة السياسية الجزائرية جملة من الإجراءات التي تراوحت بين الإجراءات الوقائية والإجراءات الصارمة من أجل محاصرة هذا الوباء والقضاء عليه في أقل وقت ممكن وبأقل الخسائر، بداية بإعلان رئيس الجمهورية عبد المجيد تبون عن وقف جميع وسائل النقل العمومي والخاص داخل المدن والو لايات وحركة القطارات، تسريح 0 \%٪ من الموظفين مع الإحتفاظ برواتبهم، تسريح النساء العاملات اللاتي لهن أطفال صغار، غلق المقاهي، المطاعم ، ضبط السوق، توقيف الملاحة الجوية والملاحة البحرية، بالإضافة إلى ضرورة الحجر الصحي لكل الوافدين للجزائر لمدة 14 يوما، وغلق المساجد وتعليق الصلاة فيها بشكل مؤقت مع الإبقاء على الآذان واستعال عبارة "الصلاة في بيوتكم" عند نهاية الآذان، و كذلك منع جميع التجمعات(6). 


\section{أولا : الإطار القانوني المنظم لإدارة الكوارث والمخاطر الكبرى يْ الجزائر:} يعتبر الإطار القانوني وبحسب مستوى جاهزيته ومو اكبته لموضوع الكوارث الطبيعية والأخطار الكبرى، من الآليات المهمة لتمديد الصلاحيات والمسؤوليات في حالة حدوث كوارث طبيعية أو أي وجود تهديدات محتملة الوقوع في شكل غخاطر كبرى.

\section{1- القانون رقم 04-20 :}

لقد تم النص من خلال المادة العاشرة من القانون 04 -20(7)على مجموع الأخطار الكبرى الخاضعة للوقاية وهي: الزلازل، الأخطار الجيولوجية، الفيضانات، الخطار المناخية، حرائق الغابات، الأخطار الصناعية و الطاقوية، الأخطار المتصلة بصحة الإنسان، الأخطار المتصلة بصحة الحيوان والنبات، أشكال التلوث الجوي أو الأرضي أو البحري أو المائي، الكوارث المترتبة على التجمعات البشرية الكبيرة(8). عرفت الجزائر عبر تاريخها المعاصر العديد من الكوارث ذات الحجم الكبير والمتوسط، وعلى إمتداد هذه الكو ارث حاولت الجزائر إلى تحسين آدائها ودورها في الحد من آثار هذه المخاطر، وقطعت اشواطا معتبرة من خلال إمتلاك إطار قانوني متين ومتناسق مع الإحتياجات والإمكانيات الوطنية. إن الوقاية من الأزمات والكوارث بشتى أنو اعها، تشمل حماية الاشخاص والممتلكات، وهي من مسؤوليات الدولة في المقام الأول، حيث يتوجب عليها تنظيم الدفاع المدني، وعمل كل هياكل الدولة، ووضع خخططات الطوارئ على كافة الإقليم (9)، حيث تحتاج إدارة الكوارث والأزمات أو مواجهتها إلى سياسة وقائية محكمة، تستمد قوتها من التجارب الماضية من خلال تحديد نقاط الخطأ والصواب في التعامل مع الأزمات و الكوارث، وهذا لن يتحقق في نظر المختصين إلا وفقا لعو امل أساسية، مثل تقييم درجة وقوة الكارثة، وتطبيق خارطة لمناطق الخطر، وتطويقها ثم القضاء على الخطر (10). 
ثم إن إستمرارية الأزمات ونموها وتجددها كان ولا يزال بمثابة التهديد الذي يمكن أن يطال خختلف المجتمعات البشرية، لذلك وجب العمل بقيادة متميزة ومبدعة وكقوة، وإعتملد ما تمتلكه من خصائص

شخصية وأخلاقية ومعرفية لمو اجهة الأزمات التي تتعرض لها الدولة(11). يقال إن الدساتير ليست فيما تقام للنوم (12) والقوانين أيضا فهي تتآكل مع الزمن، ولهذا لابد أن تتأقلم، فلابد أن تأخذ بعين الإعتبار التحولات و التطورات لأن دوامها مرهون بذلك(13).

\section{2- القانون المنظــم لأزمة كورونا ِِِ الجزائر:} بمجرد وقوع أزمة كورونا إتخذت الجزائر مايلي:

\section{إصدار المرسوم التنفيـي 20-0 7 ( الإجراءات الإداريـة ):}

أصدرت الحكومة الجزائرية المرسوم التنفيذي رقم 20-70 المؤرخ في 27 مارس 2020 حول توسيع إجراءات الحجر إلى كل الو لايات طبقا للمادة 02 من المرسوم التنفيذي حيث أن الولايات التي تخضع للحجر الجزئي أو حظر التجوال في ساعة معينة بالليل و بالنهار (14).

أ- المفهوم القانوني لحظر التجول أو الحجر بصورة عامة: يقصد بحظر التجول هو حظر حركة الناس في منطقة ما أو بلد لظروف استثنائية والتي تكون عادة ضمن مدى زمني معين، وقد تلجأ السلطات إلى فرض حظر التجول نتيجة لظروف استثنائية أو طارئة مثل الحروب، وإنتشار الأمر اض والأوبئة. إن حظر التجول من الناحية القانونية يؤثر بشكل أو بآخر على ممارسة الحقوق والحريات الفردية والجماعية، وهذا يختلف كثيراعلا شهدناه، بموجب حظر التجول الذي فرض في حالة الحصار بناء على المرسوم الرئاسي 1 9-196، حيث كلفت فيها القيادة العسكرية - الجيش الوطني الشعبي - وبموجب تعليمات صارمة لإعادة النظام بصرامة وبكل الوسائل التي تفرضها تلك الحالة حيث فرض فيها حظر التجول من منتصف الليل 
والسادسة صباحا في كل من الجزائر والرويبة والشراقة، وفي اليوم الموالي غير حظر التجول وأصبح من العاشرة ليلا إلى الخامسة صباحا(15). وقد رفعت حالة الحصار بموجب المرسوم الرئاسي 1 9-336.

\section{ب- استحداث لجنة ولائية لمجابهة فيروس كورونا:}

سنحاول المقارنة بين اللجنة المحدثة في زلزال الشلف (الأصنام) سنة 1980 واللجنة المحدثة بموجب

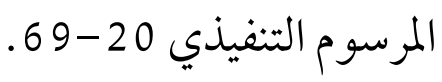

بمجرد حدوث الكارثة الطبيعية، لجأت الجزائر إلى استصدار جملة من الإجراءات الإدارية، وهي صدور مراسيم مقررة للأزمة الطبيعية ومعالجة لها، حدث زلزال في منطقة الشلف (الأصنام سابقا) في 10 أكتوبر 1980، وبتاريخ 13 أكتوبر صدر المرسوم 80-02 المحدد للإجراءات الإدارية المواكبة للظاهرة، وكذا المراسيم المنفذة للمرسوم 80-20، منها المرسوم رقم 80-254 الذي يتضمن إنشاء لجنة وطنية لتنسيق الإجراءات المتخذة لصالح المناطق المعلن عنها مناطق منكوبة، تتكون اللجنة الوطنية من:

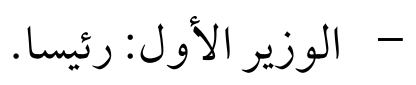

- مغوية كل من: وزير الداخلية، وزير الصحة، مسؤول الأمانة الدائمة للجنة المركزية، وزير الإعلام والصحافة، الأمين العام لوزارة الدفاع الوطني، قائد الدرك الوطني، المدير العام للأمن الوطني (16). أما اللجنة التي استحدث بموجب المرسوم التنفيذي 20-69، فقد أحدثت لجنة ولائية لمواكبة هذه الأزمة الصحية وهذا دليل على حرص القيادة السياسية على إتخاذ كافة الإجراءات القانونية والإدارية للتصدي لهذه الأزمة أو بالأحرى الكارثة الصحية. وتتكون اللجنة طبقا للمرسوم من:

$$
\text { - - - الو الي رئيسا. }
$$




$$
\begin{aligned}
& \text { - قائد بجموعة الدرك الوطني. }
\end{aligned}
$$

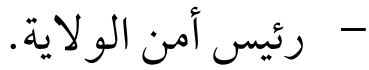

$$
\begin{aligned}
& \text { - - مثثل المديرية العامة للأمن الداخلي. } \\
& \text { - - رئيس المجلس الشعبي الولائي. } \\
& \text { - رئيس المجلس الشعبي البلدي لعاصمة الولاية. }
\end{aligned}
$$

تجدر الإشارة إلى أن هذه اللجان موجودة على مستوى كل ولايات الجمهورية الجزائرية.

ت- -لجوء الإدارة إلى وحدات الشرطة والدرك الوطني لضمان حظر تجول الاشخاص:

باستثناء استخدام القانون 1 -23 المؤرخ في 6 ديسمبر 199 و المتضمن مساهمة الجيش الوطني الشعبي في مهام حماية الأمن العمومي خارج الحالات الاستثنائية 17، والمعدل بالأمر 11-3 11 الذي يعدل ويتمم القانون 1 9-23، حيث ينص على استخدام وحدات الجيش الوطني الشعبي في حالة النكبات والكوارث الطبيعية وماية السكان ونجدنهه طبقا للمادة 202 (18). حدد المرسوم التنفيذي رقم 20-69 حظر التجول مع وجود حو اجز أمنية لأفراد الدرك الوطني والشرطة والقطاع الأمني بصورة عامة ولكنه لم يلجأ بعد وإلى غاية كتابة هذه الاسطر إلى وحدات الجيش الوطني الشعبي لضمان حظر التجول الجزئي أو الكلي.

ث- - لجوء الدولة إلى حالة الطوارئ: - مائ حالة الطوارئ هي إجراء تتخذه حكومة دولة ما في حال وجود خطر محدق يمكن من خلاله تفويض بعض الحريات الاساسية كحرية التنقل وحرية الصحافة. وقد نصت عليها المادة 4 من العهد الدولي الخاص بالحقوق المدنية والسياسية لسنة 1966. 
لجأت الجزائر إلى حالة الطوارئ في 09 فيفري 1992 كإجراء فعال لمحاربة الإرهاب، مع بدأ الدعوة إلى العصيان المدني بعد توقيف المسار الإنتخابي في ديسمبر 199 واستقالة رئيس الجمهورية الشاذلي بن جديد الذي خلفه المجلس الأعلى للدولة، بموجب المرسوم الرئاسي 92-44 المؤرخ في 09 فيفري 1992، وقد رفعت حالة الطوارئ بموجب الأمر 11 / 1 0 ، ويمكن التمبيز في هذا الصدد بين 3 مفاهيم أساسية: - - حالـة الطوارئ: هي جملة تدابير تدخل في إطار مقتضيات الأمن العام ويتم سنها أو الإعلان عنها بصفة رسمية بغية مواجهة أي خطر محدق ناتج عن المساس بالنظام العام أو أحداث قد تشكل بطبيعتها وبسبب خطور تها كارثة عامة. - الحالـة الاستثنائية: عموما تعني تلك الحالات أين يتم تعليق القانون العام ، ويمكن سنها عند المساس

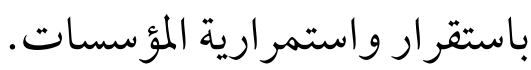
- - حالـة الحصار أو الأحكام العرفية: في زمن السلم هي جملة التدابير الأمنية يتم اتخاذها بغية الحفاظ على النظام، ويتم من خلالها تعليق وبصفة مؤقتة سريان القوانين النظامية وكذا وضع جميع السلطات في أيدي السلطة العسكرية ويتم الإعلان عنها في حال وجود خطر محدق ناتج عن حرب خارجية أو تمرد مسلح

\section{ثانيا : الإجراءات الردعية ذات الطابع العقابي (تعديل قانون العقوبات الجزائري):}

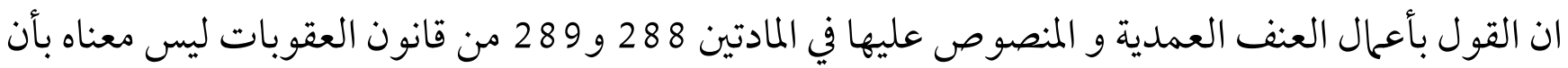
أعحال العنف غير العمدية أنها تقع بدون إرادة فانعدام الإرادة ينفي العقاب، بل إن القانون يعاقب في هذه

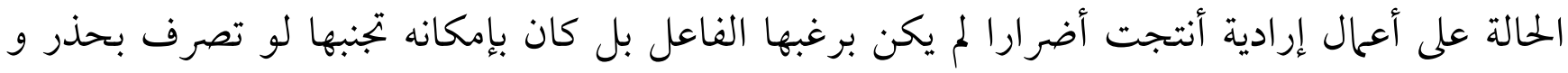
احتياط (20)

فجرم العنف تتضمن أساسا القتل و الضرب والجرح وأعحال التعدي الأخرى، وهذه الجرائم قد تكون

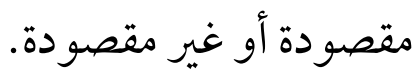


أصدر المشرع على إثر وقوع الازمة الصحية، القانون 20-60 المؤرخ في 28 أفريل 2020 و الذي يعدل الأمر رقم 66-156 المؤرخ في 8 يونيو 1966 المتضمن قانون العقوبات الجزائري، أصدر في الجريدة الرسمية العدد 25 الصادرة في 29 أفريل 2020(21)، وقد تضمنت الاحكام المقترحة تكييف هذا القانون مع التحولات الجارية في البلاد قصد التكفل بالأشكال الجلديدة للإجرام الناتجة عنها، وكذلك سد الفراغ في المنظومة القانونية في مجال تسيير الأزمات. وتشمل التعديلات تجريم الأفعال التي عرفت انتشارا كبيرا خلال السنوات الأخيرة الى درجة تهديد الأمن والاستقرار في البلاد ومن بينها ترويج أنباء كاذبة للمساس بالنظام والأمن العموميين، والتزوير للحصول على الإعانات والمساعدات المالية والمادية والعينية العمومية والإعفاءات الاجتماعية، والأفعال الماسة بنزاهة الامتحانات والمسابقات، وتعريض حياة الغير أو سلامته الجمسية للخطر، وكذلك تشديد العقوبات في جرائم الإهانة والتعدي على الامام، أو هدم و تدنيس أماكن العبادة العمومية، وكذلك دفع الحدين الأدنى و الأقصى لعقوبة العز امة المتصلة بمخالفة الأنظمة الصادرة عن الإدارة(22). تتداول الأحكام العقابية بالأزمة الصحية و الوبائية كالآتي:

\section{1 - جريهمة نشر وترويج الأخبار وأنباء تمس النظام والأمن العموميين:}

جاءت المادة 196 مكرر من قانون العقوبات الجزائر المعدل برفع الحد الأدنى و الحد الأقصى للعقوبة و كذلك رفع قيمة العزامة المالية المقصودة لهذه الجريمة كالتالي: عقوبة الحبس من سنة الى 3 سنوات. و العزامة من و ترصد هذه العقوبة لكل من يقوم بنشر أو يروج عمدا بأية وسيلة كانت أخبار أو أنباء كاذبة أو مغرضة بين الجمهور يكون من شأنها المساس بالأمن العمومي أو النظام العام. 


\section{2- جريهــة التزويـر للحصـول علـى الإعـانـات والمسـاعدات العموميــة والإعفــاءات}

الاجتهماعية:

نصت على هذه الجريمة المادة 253 فقرة 10 من قانون العقوبات و قد عاقبت مرتكب الفعل عقوبة الحبس من سنة المى 3 سنوات و غرامة مالية من 100.000 دج الى 300.000 دج كل من يتحصل على إعانات أو مساعدات مالية أو مادية أو عينية من الدولة أو الجماعات الإقليمية أو أي هيئة عمومية أخرى أو على إعفاءات في المجال الاجتماعي ، عن طريق التزوير في الوثائق أو التصريح الكاذب أو باستعال معلومات خاطئة أو ناقصة. و يعاقب بنفس العقوبة كل من يستمر في أخذ هذه الإعانات حتى و لو زالت الظروف التي من شأهها رصت هذه الاعانة المالية أو الاقتصادية. ويعاقب كل يمول هذه الإعانات الى وجهة أخرى غير مستحقة فإنه يعاقب بالحبس من سنتين الى 3 سنوات و بغرامة من 200.0000000 د300 دج و تضاعف العقوبة في حالة العود، حيث تخضع العقوبة و حالات العود لنفس الشروط العامة و الاحكام العامة لنظرية الجريمة. 23 كما ترصد لمرتكب الجريمة و طبقا للمادة 253 مكرر2 و إضافة الم العقوبات الاصلية العقوبات التكميلية

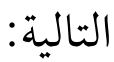

1 - رد الإعانات و المساعدة المالية أو المادية أو الفنية أو الإعفاءات المتحصل عليها بغير وجه حق، أو رد قيمتها. إضافة الى حرمانه من حق أو أكثر المنصوص عليها في المادة 0 فقرة 01 من قانون العقوبات الجزائرية24. كما يعاقب على المحاولة في هذه الجريمة بنفس عقوبة الجريمة التامة 5 . 2- القتل الخطأ و الجرح الخطأ و تعريض حياة الغير و سلامته الجمدية للخطر، قام المشرع الجزائري بتعديل نص المادة 08 من الأمر رقم 66-156 المتضمن قانون العقوبات بالمادة 290 مكرر، حيث أسس المشرع الجزائري حكمه الوارد في المادة 290 مكرر بالحبس من 06 أشهر الى سنة و بغرامة من 000000 دج الى 200.000 دج كل من يعرض حياة الغير أو سلامته الجمدية مباشرة للخطر المتعمد و النسبي لواجب من 
واجبات الاحتياط و السلامة التي يفرضها القانون أو التنظيم وتكون العقوبة الحبس من 03 سنوات إلى

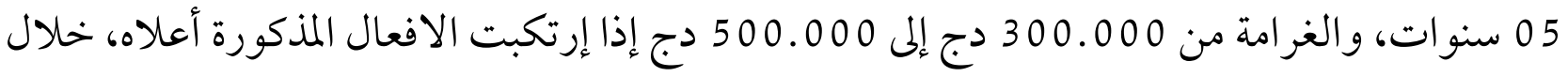
فترات الحجر الصحي أو خلال وقوع كارثة طبيعية أو بيولو جية أو تكنلوجية أو غيرها من الكوارث. كا قررت المادة 459 من ذات القانون و التي عدلت المادة 9 من الامر 66-156 المتضمن قانون العقوبات عقوبة لكل من يخالف الحجر الصحي وهي الغرامة من 10.000 دج إلى 20.000 دج وييوز أن يعاقب ايضا بالحبس لمدة 03 ايام على الأكثر، وتكون هذه العقوبة مقررة لكمل من يخالف المراسيم والقرارات المتخذة قانونا من طرف السلطة الإدارية إذا لم تكن الجر ائم الواردة بها معاقبا عليها بنصوص خاصة. كما قررت المادة 459 مكرر من ذات القانون و التي عدلت المادة 10 من الامر 66-156 المتضمن قانون العقوبات: بأن الدعوى العمومية المقررة للجريمة المنصوص عليها في المادة 459 تنتفي بدفع غرامة جزافية يساوي مبلغها 10.000 دج أما بالنسبة للجانب الاجرائي فهو كالتالي: يمنح مرتكب المخالفة أجل 10 أيام من تاريخ الاخطار بالمخالفة لدفع مبلغ الغرامة لذى قابض الضرائب

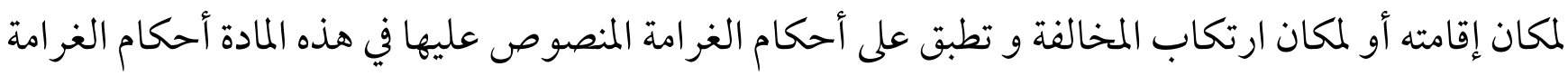
الجزافية المنصوص عليها في قانون الإجراءات الجزائية 27 المادة 11 من قانون 20-06 و التي عدلت المادة 465 من الأمر 66-156 المعدل لقانون العقوبات و التي تخص العائد في مواد المخالفات: وهو الحبس الذي تصل مدته 10 أيام وغرامة قد تصل الى 32.000دج في حالة العَود بالنسبة لمخالفات

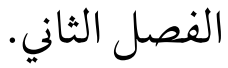
والحبس الذي تصل مدته الى 5 أيام وبغرامة تصل الى 30.000دج في حالة العود بالنسبة لمخالفات الفصل

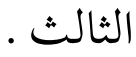




\section{خاتهــــــــة:}

من خلال ما سبق يمكن تصنيف علم إدارة الأزمات كواحد من العلوم الإنسانية الحديثة، الذي ازدادت أهميته في العصر الحالي. وهو علم إدارة توازنات القوى ورصد حركتها وإتجاهاتها، وهو أيضا علم المستقبل، وعلم التكيف مع المتغيرات، وعلم تحريك الثوابت وقوى الفعل في المحالات الإنسانية كافة، إنه علم المستقبل متصل بكافة العلوم الإنسانية.

إن دراسة إدارة الأزمات قد تطورت وأصبحت بجالا مشتركا لإهتحام وعمل الباحثين والخبراء من تخصصات علمية يختلفة تجمع كافة فروع العلوم الإنسانية والطبيعية، وعلى الرغم من أن أصحاب كل تخصص يتعاملون مع إدارة الأزمة كل من زاوية إهتحامه و خلفيته النظرية وخبراته العلمية، إلا أن هناك نقاطا كثيرة الإلتقاء و التعاون و العمل المشترك فرضتها الطبيعة النوعية المركبة لمعظم الأزمات ، الأمر الذي فرض ضرورة تكوين فريق عمل من مختلف التخصصات والخبرات لمواجهة الأزمات وحسن التعامل معها. مما سبق نستنتج ما يلي: 1 - تعاملت الجزائر مع أزمة كورونا من الناحية القانونية بإصدارها لقوانين إدارية و قو انين عقابية. 2- إن الأزمات عادة ما تنتهي بالتو جه المحكم و السيطرة عليها. 3- إن الأزمات تخلق نوعا من حالة اللاإستقرار داخل المجتمع و تكون آثارها في كافة المجالات .

$$
\text { و نقترح ما يلي: }
$$

1 - إن تعديل قانون العقوبات الجزائري و تضمينه لأحكام جزائية رادعة للأزمة التي تعتمد على وعي

$$
\text { المو اطن بحجم الخططر . الم. }
$$

2- إن إصدار القوانين الإدارية المتراوحة بين الحجر الكلي و الحجر الجزئي أصبحت تشكل معيارا لمدى

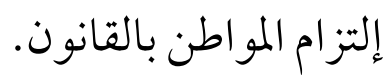


3- إن تمييز منظمة الصحة العالمية لمفهومي الجائحة و الوباء ، هو تمييز قانوني أكثر منه صحي بإعتبار أن كلاهما ذو أصل فيروسي سريع الانتشار و يبقى الرهان دائما على وعي المواطن.

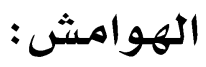

(1) عبد الله الزيوت، منتصر، عبد الله الزيوت، معتصم، إدارة الأزمة الإقتصادية في ظل وباء كورونا وفق مبادئ الاقتصاد الإسلامي،

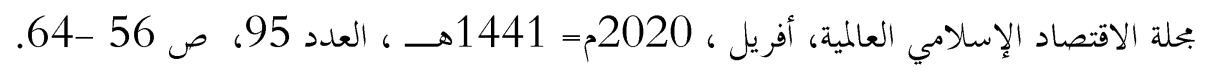

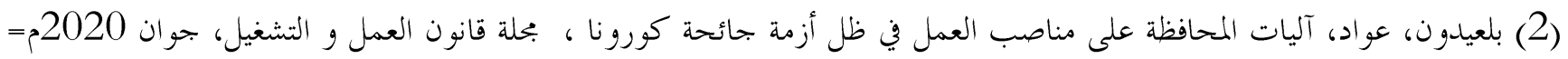

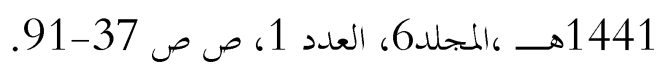

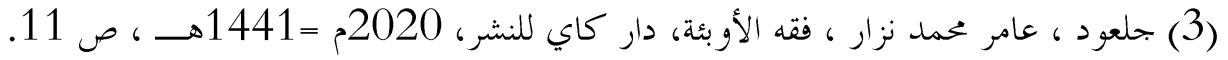

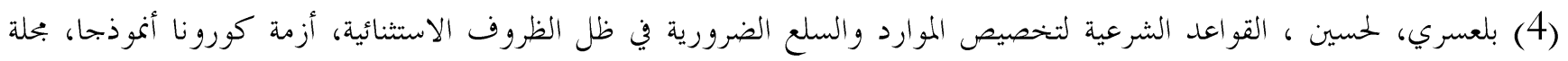

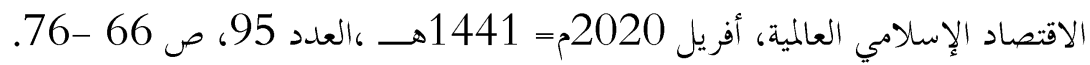

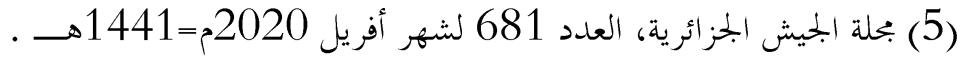

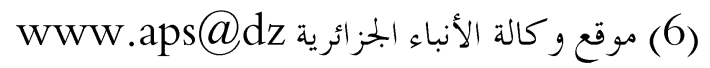

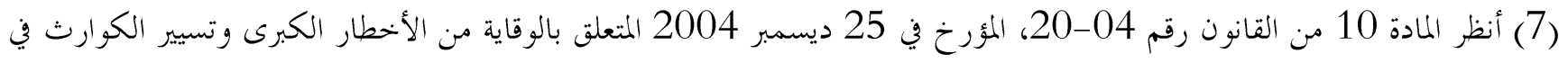

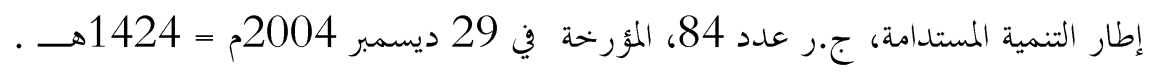

(8) غربي ، عزوز ، إدارة الكوارث والمخاطر الكبرى في الجزائر على ضوء القانون 04-20، بحلة الدراسات والبحوث القانونية ،

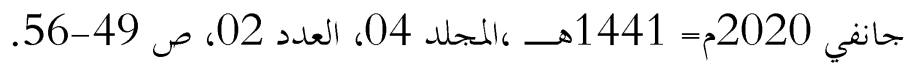

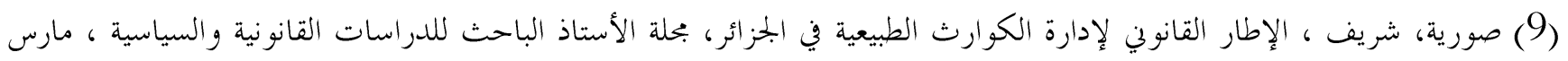

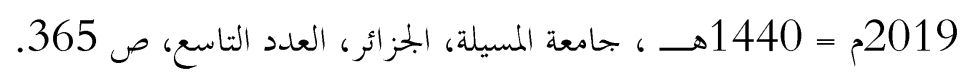

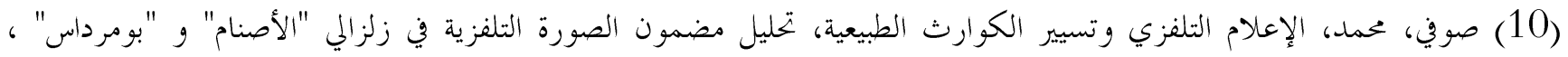

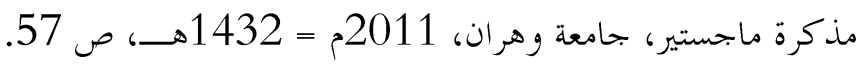

(11) بغدادي، فيصل، دور القيادة في إدارة الأزمات في المنطقة، مذكرة ماستر، كلية الحقوق، جامعة المسيلة، 2014م = 1435هـ،

F.Royer collard cité par j.Gicquel, Essai sur la pratique de la 5éme république, Paris, (12)

$$
\text { L.G.D.J, 1968, P28. }
$$

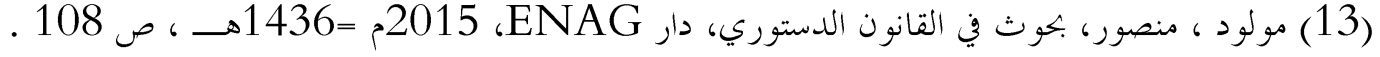

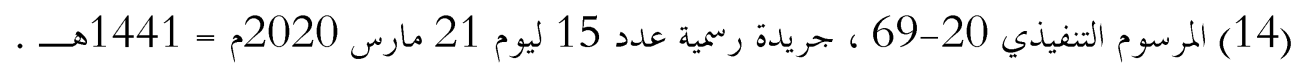


(15) بوالشعير، سعيد ، سلطات رئيس الجمهورية في الظروف الغير العادية، المجلة الجزائرية للعلوم القانونية والاقتصادية السياسية،

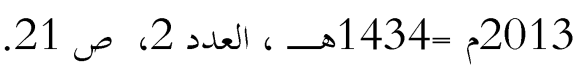

(16) مداخلة الدكتورة عاثشة عبد الحميد في ملتقى وطني حول الأزمات والكوارث بحامعة 08 ماي 1945 بقالمة، يوم 16 ديسمبر

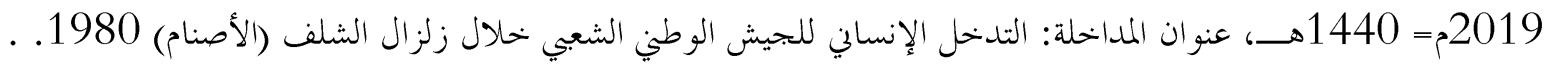

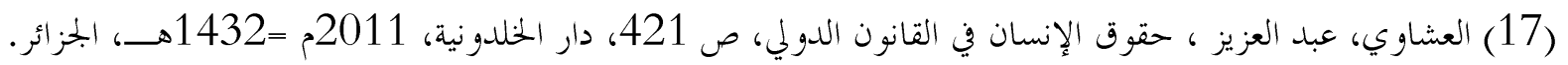

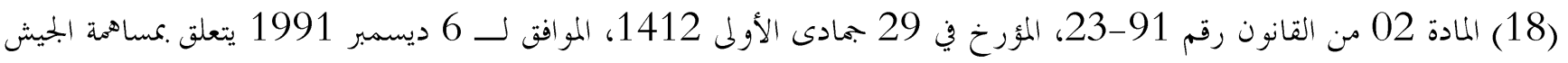

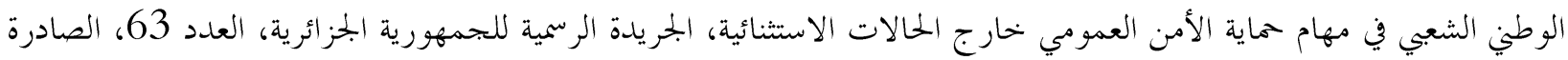

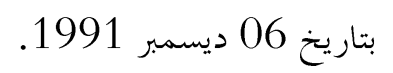
(19) جبار، صلاح الدين، القضاء العسكري في التشريع الجزائريو القانون المقارن، دط، ص 277 2017، دار الخلدونية،

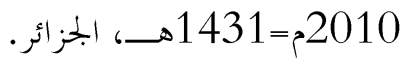

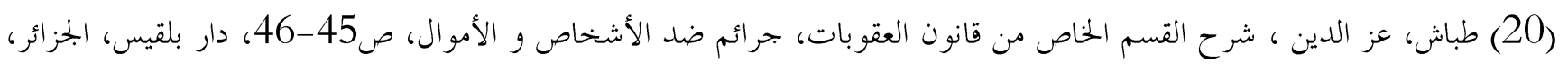
2018م =1439هـ

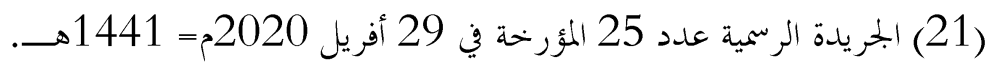

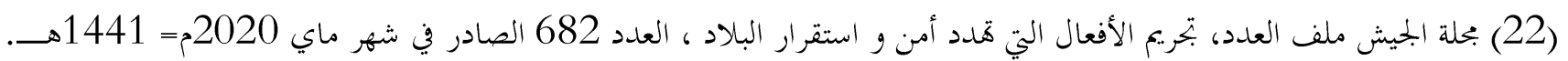

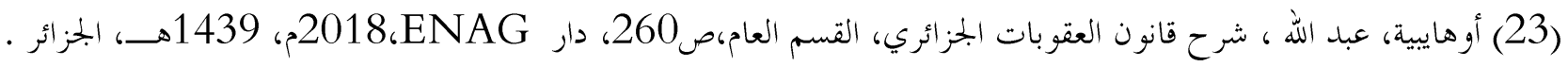

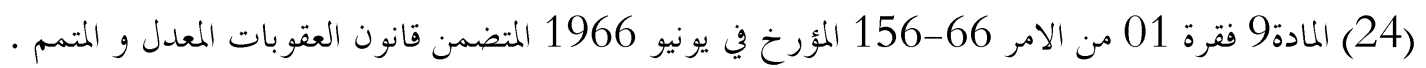

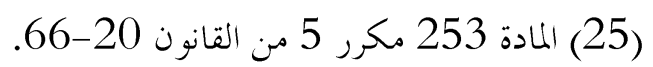

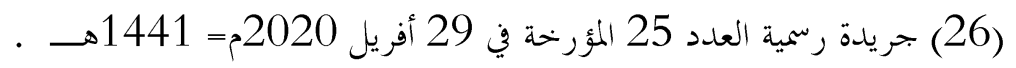

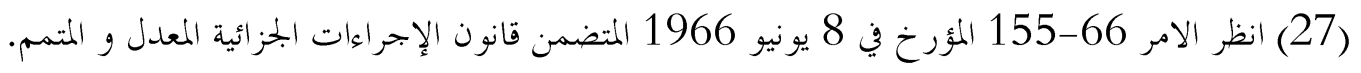

